\title{
Gravitational and Magnetic Separation in Self-Assembled Clay-Ferrofluid Nanocomposites
}

\author{
F.L.O. Paula \\ Institute of Physics, University of Brasília, Brasília, Brazil \\ G.J. da Silva, R. Aquino, and J. Depeyrot \\ Institute of Physics. University of Brasília, Brasília, Brazil \\ J.O. Fossum \\ Department of Physics, Norwegian University of Science and Technology, Trondheim, Norway \\ K.D. Knudsen and G. Helgesen \\ Physics Department, Institute for Energy Technology, Kjeller, Norway \\ F.A. Tourinho \\ Complex Fluids Group, Institute of Chemistry, University of Brasília, Brasília, Brazil
}

(Received on 1 September, 2008)

\begin{abstract}
We report on experimental observations of self-assemblies in colloidal dispersions of clay nanoplatelets and magnetic nanoparticles. Visual observations have been combined with small angle X-ray scattering (SAXS) in the study of several composites at a fixed clay concentration in the dilute regime, and varying ferrofluid concentrations. Our visual observations which encompass macroscopic separation in gravitational- and magnetic field, indicate that all samples present a concentrated phase and a diluted one. SAXS data obtained from each phase are consistent with the interpretation that the scattering contribution from the clay nano-platelets in the samples can be neglected in comparison with the magnetic particle contribution. The analysis of the scattered intensity is performed combining two models, one based on the global scattering function and the other allowing the extraction of the structure factor of the mixtures. The parameters of the size distribution of magnetic nanoparticles determined by both methods are in good agreement. The structure factor of the mixtures shows that on a local scale, the mixtures behave like a gas of isolated magnetic nanoparticles. It also indicates the presence of interactions between magnetic nanoparticles mediated by the presence of Laponite platelets. Such interactions could be attributed with a progressive partial phase separation between spheres and discs rather than to the formation of dense aggregates.
\end{abstract}

Keywords: VER

\section{Introduction}

Self-assembly of nanoparticles and the use of such particles in composite materials are currently very active areas within chemistry, physics, and nanoscience [1]. Many selfassembled systems under investigation are colloidal particle systems, such as suspensions of carbon nanotubes, clays, ferrofluids etc. [1-3]. To predict and monitor the thermodynamic stability of dispersions and to associate theoretical models with the experimental results represents a challenge in colloidal science. Several technological and industrial applications require stable colloids, and this is directly related to the process of self-assembly and phase separation. Colloidal stability and self-assembly is very complex and involves several kinds of particle interactions [4], van der Waals and magnetic dipole-dipole interactions and repulsive electrostatic interaction. Considering the solvent as a continuous medium, it is possible to make analogies between colloidal and atomic systems because the interparticle interaction potential displays the same overall shape for both systems [5]. With this point of view, liquid phases, gas phases and solid phases can be observed in colloidal dispersions. However unlike atomic systems, inter-particle interactions in complex fluids made from self-assembled colloidal nanoparticles can be tuned by experimental parameters such as ionic strength [6], temperature, colloidal $\mathrm{pH}$, density and size of nanoparticles [6-8], magnetic field $[3,9,10]$ or electrical field $[11,12]$.
In the present case, we report on experimental investigations of composite mixtures of two such complex particle systems; i.e. composites of Laponite clay nanoplatelets [13] and magnetic nanoparticles [14]. Dispersions of discotic nanoparticles of Laponite present aging properties and a well known rheological state diagram with an isotropic-nematic transition. Ferrofluids are colloidal dispersions of ferrite nanoparticles and combine liquid properties and susceptibility to an applied magnetic field. By associating both aqueous colloidal systems, one may thus obtain a magneto-rheological system. The present work expands and elaborates in depth on a previous preliminary report on these systems [15].

Each of the two ingredient aqueous colloidal systems, i.e. clay and ferrofluid, have their own phase behavior as a function of several parameters and are in the present case strongly dependent on the electrostatic interactions [4]. The chemical synthesis [16] and the colloidal stability [17] of magnetic nanoparticles in aqueous media are relatively well known, and their phase behavior as a function of ionic strength and temperature has been studied using small angle neutron scattering.

Laponite $[10,19]$ is a widely studied synthetic clay that belongs to the family of swelling 2:1 clays [2,20]. The colloidal phase diagram of aqueous Laponite suspensions as a function of ionic strength and concentration has been investigated [10] and displays four different regions (phases): isotropic liquid, isotropic gel, nematic gel and flocculation.

Previously maghemite magnetic nanoparticles have been 
incorporated as a probe into clay dispersions in order to characterize their spatial repartition and micro-rheological behavior [21]. In the present work, we prepare a Laponite clay dispersion $(0.1 \mathrm{wt} \%)$ in the isotropic liquid phase, and combine this with citrated ferrofluid based on cobalt ferrite nanoparticles at several concentrations in order to obtain composite products. We report here on experimental investigations and results from visual observations combined with small angle $\mathrm{X}$-ray scattering experiments.

In the following sections, we first describe in detail the aqueous suspensions of Laponite nanoplatelets, the synthesis of magnetic nanoparticles and the elaboration of composite mixtures. Then, visual observations of the composites and small angle scattering experiments are presented and followed by a description and discussion of the experimental observations.

\section{Materials and Methods}

\section{Aqueous suspensions of Laponite nano-platelets}

Laponite is a synthetic clay with general formula $\mathrm{Na}^{+0.7}$ $\left[\mathrm{Si}_{8} \mathrm{Mg}_{5.5} \mathrm{Li}_{0.3} \mathrm{H}_{4} \mathrm{O}_{24}\right]^{-0.7}$, diameter around $25 \mathrm{~nm}$ and thickness of $1 \mathrm{~nm}$, and nearly monodisperse. The chemical $\mathrm{pH}$ stability range of Laponite platelet solutions is narrow: below $\mathrm{pH} 9$ the magnesium ions dissolve in solution, and above $\mathrm{pH} 10$ the dissolution of silica is observed [22]. The structural charge of the surface of the platelets is negative. The edge charge depends on the acid-base behavior of the $\mathrm{Si}-$ $\mathrm{OH}$ and $\mathrm{Mg}-\mathrm{OH}$ amphoteric hydroxyl groups which are the main species on the edge. Figure 1a shows a Laponite particle and its crystallographic structure. Laponite powder with high chemical purity was purchased from Laporte Absorbents (UK). The Laponite stock solution was prepared in a sodium citrate solution with trisodium citrate $\left(\mathrm{Na}_{3} \mathrm{Cit}\right)=2.5 \times 10^{-3}$ $\mathrm{mol} / \mathrm{l}$ and vigorously stirred for $48 \mathrm{~h}$.

\section{Aqueous suspensions of synthesized magnetic nanoparticles}

Cobalt ferrite nanoparticles were obtained using a hydrothermal coprecipitating aqueous solution of a $\mathrm{CoCl}_{2}-$ $\mathrm{FeCl}_{3}$ mixture in alkaline medium [16]. After the coprecipitation step these particles were conveniently peptized in an acidic medium by adjustment of the ionic strength resulting in an aqueous stable sol of high quality at $\mathrm{pH} \sim 2$. Addition of $\mathrm{Na}_{3} \mathrm{Cit}$ to the solution allows coating of the particles with citrate ions for dispersion in aqueous solution at $\mathrm{pH} \sim$ 10. Figure $1 \mathrm{~b}$ displays the crystallographic structure of Cobalt ferrite nanoparticles.

In the present case, the mean size of our nanoparticles was determined through X-ray powder diffractograms. According to the Scherrer equation and using the half width of the strongest diffraction peak it leads to an average size $14.0 \mathrm{~nm}$. TEM allowed also the morphology and the size distribution of the nanoparticles to be characterized. The size distribution is usually described by a log-normal law:

$$
P(d)=\frac{1}{\sqrt{2 \pi} d \sigma} \exp \left(-\frac{\ln ^{2}\left(d / d_{0}\right)}{2 \sigma^{2}}\right),
$$
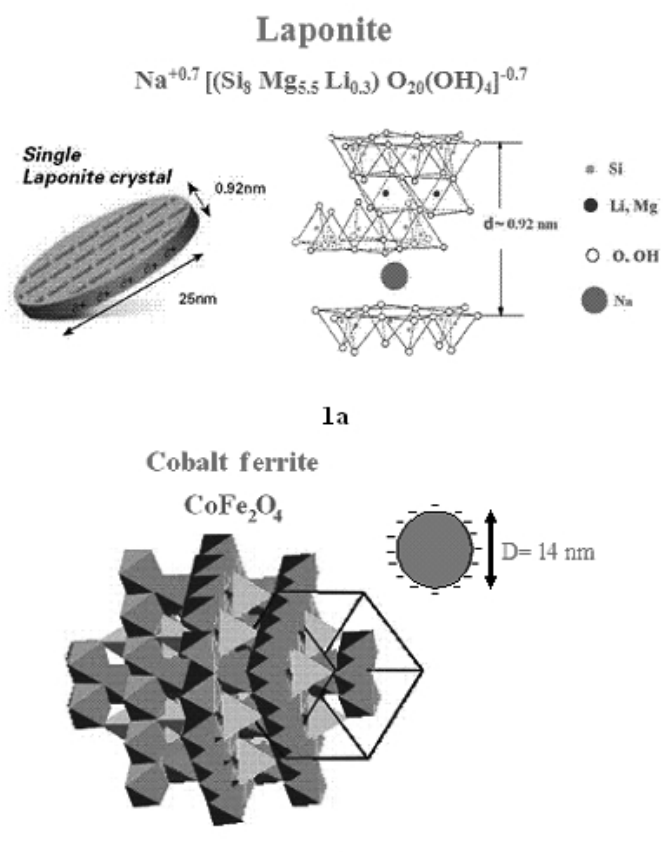

$1 \mathbf{b}$

FIG. 1: Characteristic shape, dimensions, crystallographic structure and chemical formula for a Laponite particle a) and a Cobalt ferrite nanoparticle b).

where $d_{0}$ is the characteristic size of the distribution and $\sigma$ the polydispersity index. From TEM pictures a sampling of about 500 individual particles was analyzed and the size histogram was obtained giving $d_{0}=11.7 \mathrm{~nm}$ and $\sigma=0.24$.

\section{Composite mixtures}

In order to obtain a stable isotropic liquid dispersion of magnetic nanoparticles and Laponite platelets it is necessary to adjust the $\mathrm{pH}$ and ionic strength of each precursor colloid. On account of the citrate adsorption equilibrium at the magnetic nanoparticle surface the ionic strength of the mixture is imposed by the sodium citrate concentration and fixed, by dilution, to the required value. As described above, the Laponite suspensions must have $\mathrm{pH}$ between 9 and 10 in order to avoid platelet degradation. In the case of citrate ferrofluids, the $\mathrm{pH}$ stability interval ranges from 4 to 10 . Since the $\mathrm{pH}$ and ionic strength regions in which it is possible to obtain a stable dispersion is narrow, we have fixed the $\mathrm{pH}$ to 10 and the ionic strength to $\mathrm{Na}_{3} \mathrm{Cit}=2.5 \times 10^{-3} \mathrm{~mol} / \mathrm{l}$ in all mixtures. This $\mathrm{Na}_{3} \mathrm{Cit}$ concentration is equivalent to an ionic strength $I=1 / 2 \sum_{i} c_{i} z_{i}^{2}$ of $1.5 \times 10-2 \mathrm{~mol} / 1$ ( $\mathrm{c}_{i}$ is the concentration of the species $i$ and $z_{i}$ is the valency). It corresponds to the regime of ionic strength where the fluid-solid transition of Laponite suspensions is shifted toward low volume fraction as the ionic strength increases. Then in order to obtain an isotropic liquid mixture the volume fraction of Laponite was kept constant and equal to $0.1 \% \mathrm{w} / \mathrm{w}$ and the volume fraction $(\phi)$ of magnetic nanoparticles was varied with more than a factor of 10 , from $0.010 \%$ to $0.25 \% \mathrm{w} / \mathrm{w}$. The samples were obtained by adding the citrated magnetic fluid to the 
$0.1 \% \mathrm{w} / \mathrm{w}$ Laponite stock solution and stirring vigorously for $10 \mathrm{~min}$. All samples were degassed to prevent any formation of $\mathrm{CO}_{3}^{-}$and stored for about 1 month before the scattering experiments.

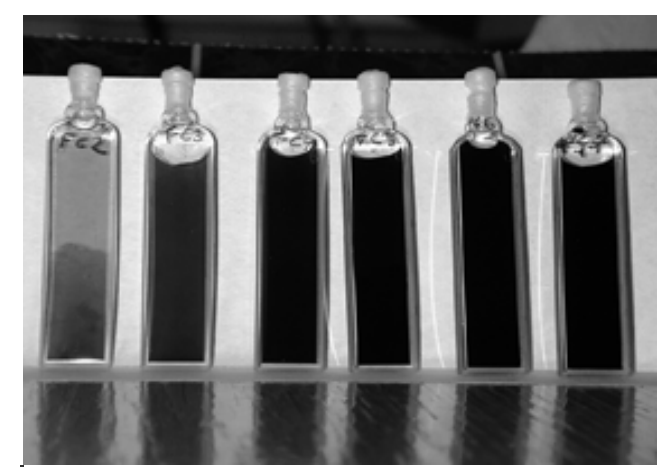

\section{FC2 FC3 FC4 FC5 FC6 FC7}

FIG. 2: Investigated samples of composite Ferroclay (FC), labeled and numbered from FC2 to FC7. Note that $\mathrm{CoFe}_{2} \mathrm{O}_{4}$ nanoparticles optically absorb in red and that Laponite nanoplatelets do not.

Figure 2 displays the investigated composite (FC) samples labeled from FC2 to FC7 with increasing content of magnetic nanoparticles. For all samples, there is a concentrated region (lower part) and a diluted region (upper part). This is most clearly evidenced for the FC2 sample, even if, in the lower part of the bottle, concentrated domains mixed with more diluted regions can be seen. The characteristics of the studied samples are collected in Table 1 which also gives the details of pure ferrofluid (FF) and pure Laponite samples (Lp). The table lists their respective concentrations of nanoparticles in grains $/ \mathrm{cm}^{3}$, their corresponding volume fraction of nanoparticles $\phi(\%)$, the ratio between the number of platelets and the number of ferrite nanoparticles, as well as the ratio between the volumes of each material.

\begin{tabular}{|c|c|c|c|c|}
\hline Samples & $\begin{array}{c}\text { Particles Concentration } \\
\left(10^{14} \text { grains } / \mathrm{cm}^{3}\right)\end{array}$ & $\phi(\%)$ & $\begin{array}{c}\text { Number ratio } \\
L p / F F\end{array}$ & $\begin{array}{c}\text { Volume ratio } \\
L p / F F\end{array}$ \\
\hline FF & $4.0 \times 10^{2}$ & 1 & - & - \\
\hline Laponite & 11.0 & 0.1 & - & - \\
\hline FC2 & 0.9 & 0.010 & 11.91 & 3.37 \\
\hline FC3 & 2.3 & 0.025 & 4.76 & 1.34 \\
\hline FC4 & 4.7 & 0.050 & 2.38 & 0.67 \\
\hline FC5 & 7.0 & 0.075 & 1.58 & 0.44 \\
\hline FC6 & 9.3 & 0.100 & 1.19 & 0.33 \\
\hline FC7 & 23.0 & 0.250 & 0.47 & 0.13 \\
\hline
\end{tabular}

Table 1: Content in ferrite nanoparticle and Laponite nanoparticles in the samples studied.

\section{Visual observations:}

In order to investigate the behavior of this composite samples under an applied magnetic field, one of the samples (FC3) was loaded into a $1 \mathrm{~mm}$ quartz cell and placed in contact with a discoid rare earth permanent magnet of approximately
1.2 Tesla, which induces a strong magnetic field gradient. Visual microscopy observations were recorded at regularly intervals of time, and pictures were taken for situations with and without a magnetic field gradient.

\section{Small angle X-ray scattering (SAXS):}

The SAXS experiment was done at beamline D11A-SAXS at the Laboratorio Nacional de Luz Sincrotron (LNLS) in Campinas, Brazil (energy range 6-12 keV). All data were collected at a wavelength, $\lambda$ of $1.756 \AA$ with a sample-to-image plate detector distance of $1.5 \mathrm{~m}$ resulting in a covered $q$ range from about 0.01 to $0.2 \AA^{-1}$, where $q=(4 \pi / \lambda) \sin \theta$ is the modulus of the scattering vector, and $2 \theta$ is the scattering angle. The samples were contained in metricconverter in $1 \mathrm{~mm}$ quartz capillaries. Data treatment was performed using the software FIT2D (free download from ESRF: www.esrf.eu) with the usual corrections included in its routine integration of the two-dimensional SAXS pattern. The output of this software provides the corrected intensities ready for subtraction of the scattering from the citrated solution and the quartz capillary. The scattering length densities of $\mathrm{CoFe}_{2} \mathrm{O}_{4}$ nanoparticles, Laponite platelets, water and sodium citrate are 4.35, $2.29,0.94$ and $1.66 \times 10^{11} \mathrm{~cm}^{-2}$ respectively.

\section{Theoretical considerations}

\section{Scattered intensity}

The scattered intensity from a dispersion of single and centrosymmetrical objects, each of average volume $V$, can be written as $I(q)=n V^{2} \Delta \rho^{2}|F(q)|^{2} S(q)$, where $n$ is the number of scattering objects per volume unit $\left(\mathrm{cm}^{-3}\right)$ of solution, $\Delta \rho$ is the contrast between the particles and the solvent, $P(q)=$ $|F(q)|^{2}$ is the particle form factor and $S(q)$ is the structure factor of the solution. For a dilute dispersion of isolated scattering objects, $S(q)=1$. In this case, at sufficiently low $q$, the scattering may be described by the Guinier law which allows to determine the Guinier scattering object radius of gyration $\left(R_{g}^{G}\right)$ by the expression $\left.I(q)=I(0) \exp \left(-q^{2}\left(R_{g}^{G}\right)^{2} / 3\right)\right)$. At high values of $q$, i.e. when $q>>1 / R_{g}^{G}$, the scattering curve reflects the characteristics of the interface between the scattering object and the solvent (Porod region). In the case of non-interacting randomly oriented platelets $(h<<D$, where $\mathrm{h}$ is the platelet thickness and D is the platelet diameter), the measured scattered intensity scales as $q^{-2}$ in the intermediate range (See Ref. 15). For the case of spherical non-interacting particles, the scattering intensity in the Porod regime scales as $q^{-4}$.

For a polydisperse set of scattering objects, the size distribution must be taken into account and, using scattering data, the size distribution function can be obtained by a numerical routine.

In the following, we briefly describe the two models that are combined here to analyze the scattering curves, on the basis of assuming that the scattering of clay is negligible. This assumption is firstly justified since 
$\Delta_{\text {Ferrite }}^{2} V_{\text {Ferrite }}^{2} / \Delta \rho_{\text {lapo }}^{2} V_{\text {lapo }}^{2}$ is about $10^{2}$; then, the $\mathrm{I}(0)$ scattering of ferrite nanoparticles should be superior than the $\mathrm{I}(0)$ scattering of laponite platelets by more than one order of magnitude for FC2 and two orders of magnitude for samples FC6 and FC7. Moreover, we have already shown in reference 15 , where we present SAXS scattering from a $1 \%$ pure citrated ferrofluid solution and a $0,1 \mathrm{wt} \%$ pure clay dispersion, that the scattering of our Laponite-ferrofluid composite sample FC3 is mainly due to the magnetic nanoparticles since we observe about one order of magnitude lower SAXS scattering from pure Laponite as compared to pure ferrofluid for our concentrations.

\section{Global scattering function, the unified equation}

Here, we use a global scattering function proposed by G. Beaucage [23] which unifies the Guinier and Porod local laws in only one function which describes the form factor of a scattering object in terms of radius of gyration and interfaces in the medium. This function writes:

$$
I(q)=G \exp \left(\frac{-q^{2} R_{g}^{2}}{3}\right)+\frac{B}{q_{o}^{n}}+y_{0},
$$

with $q_{o}=q /\left[\operatorname{erf}\left(q R_{g} / \sqrt{6}\right)\right]^{3}$, where $\operatorname{erf}(x)$ is an error function, $G$ is the Guinier pre-factor proportional to the concentration of scattering objects and to the square of the volume, $B$ is a constant pre-factor specific to the kind of power law obtained for high $q$ values and $v_{0}$ is a term introduced in order to take into account the incoherent scattering. This global scattering function describes both regimes at low and high values of the scattering vector $q$. In general, the value of the exponent $n$ is between 3 to 4 in the case of fractal surfaces, smaller than 3 for fractal masses and larger than 4 for diffuse interfaces [17, 24-26]. These models have been successfully used to characterize a disordered cluster structure [28], a fractal mass of particles [29] as well as to distinguish between individual polydisperse particles and aggregates [30]. Polydispersity of the particles serves to increase the dimensionless ratio $B R_{g}^{4} / G$ associated with the size Polydispersity Index (PDI) of the scattering object. For a system of polydisperse spheres described by a log-normal size distribution, the parameters $R_{0}$ (the characteristic radius of the distribution) and $\sigma$ can be obtained directly by the unified fit parameters, through the spherically normalized PDI [30] and $B R_{g}^{4} /(1.62 G)$ :

$$
R_{0}=\sqrt{\frac{5}{3}} R_{g} \exp \left(-7 \sigma^{2}\right) \text { and } \sigma=\left(\frac{\ln P D I}{12}\right)^{1 / 2} .
$$

Using the definition of $P D I$ we can rewrite the global scattering function as:

$$
\begin{aligned}
I(q)= & G\left\{\exp \left(\frac{-q^{2} R_{g}^{2}}{3}\right)+\right. \\
& \left.+\left(\frac{1.62 P D I}{R_{g}^{4}}\right) \frac{\left\{\operatorname{erf}\left(q R_{g}^{2} / 6^{1 / 2}\right)^{3}\right\}^{n}}{q^{n}}\right\} y_{0} .
\end{aligned}
$$

\section{Extraction of the structure factor}

For monodisperse spheres of diameter $d$, the form factor is the square modulus of [31]:

$$
F_{d}(q)=3 \frac{\sin (q d / 2)-\cos (q d / 2)}{(q d / 2)^{3}} .
$$

The size distribution must be taken into account by considering a superposition of contributions of each different particle weighted by the square of the particle volume. We can therefore write the form factor as:

$$
P(q)=\frac{\int_{0}^{\infty}\left|F_{d}(q)\right|^{2} P(d) d^{6} \mathrm{~d} d}{\int_{0}^{\infty} P(d) d^{6} \mathrm{~d} d} .
$$

If the clay scattering is negligible, $I(q) \sim P(q) S_{\text {mixtures }}(q)$. As $P(q)$ has been independently measured on a pure solution of isolated magnetic nanoparticles [15], one can easily calculate the structure factor of the mixtures $S_{\text {mixtures }}(q)$.

\section{Results and discussion}

2D SAXS scattering patterns were collected from the samples presented in Table 1. Subsequently, the two-dimensional images were integrated in order to obtain one-dimensional scattering curves as a function of scattering vector magnitude, $q$. These one-dimensional scattering curves that have been analyzed below. The data were obtained for each of the two gravity-induced regions indicated in Figure 2, that are separated into an "upper" and a "lower" part. These measurements were performed at equilibrium conditions, in the absence of external magnetic field.

In our preliminary report on these systems [15], we showed that equation 4 describes very well both the SAXS and SANS $I(q)$ scattering curves obtained from our pure Laponite dispersion and pure citrated ferrofluid solution. For the $0.1 \%$ wt dilute dispersion of Laponite nanoplatelets, we observed that the scattering intensity is low and we concluded that the SAXS scattering power of our Laponite dispersion scale as $q^{-2}$ in agreement with previous observations [32]. For the pure citrated ferrofluid solution, the deduced values of the lognormal size distribution parameters were in good agreement with Rietveld refinement of the X-ray powder diffraction pattern of magnetic nanoparticles. We have furthermore observed that the scattering is proportional to the ferrofluid particle concentration, indicating that we are working in a non-interaction regime as far as the pure ferrofluid is concerned. For small values of $q$, typically $q<0.01 \AA^{-1}$, the $\log -\log$ plots from our pure ferrofluid samples show a plateau, indicating the absence of large aggregates and no significant interactions between scattering objects. For larger $q$ values, the log-log scattering intensity scales with $q^{-4}$, as will be the case for 3-D objects with a sharply defined smooth interfaces. Moreover, using equation 6, the nanoparticles form factor can also be determined.

Figures $3 \mathrm{a}$ and $3 \mathrm{~b}$ presents the SAXS scattering curves obtained for both composite phases and for different samples at various ferrofluid concentrations as described in Table 1. It is observed that the high-q region scales as $q^{-4}$ 

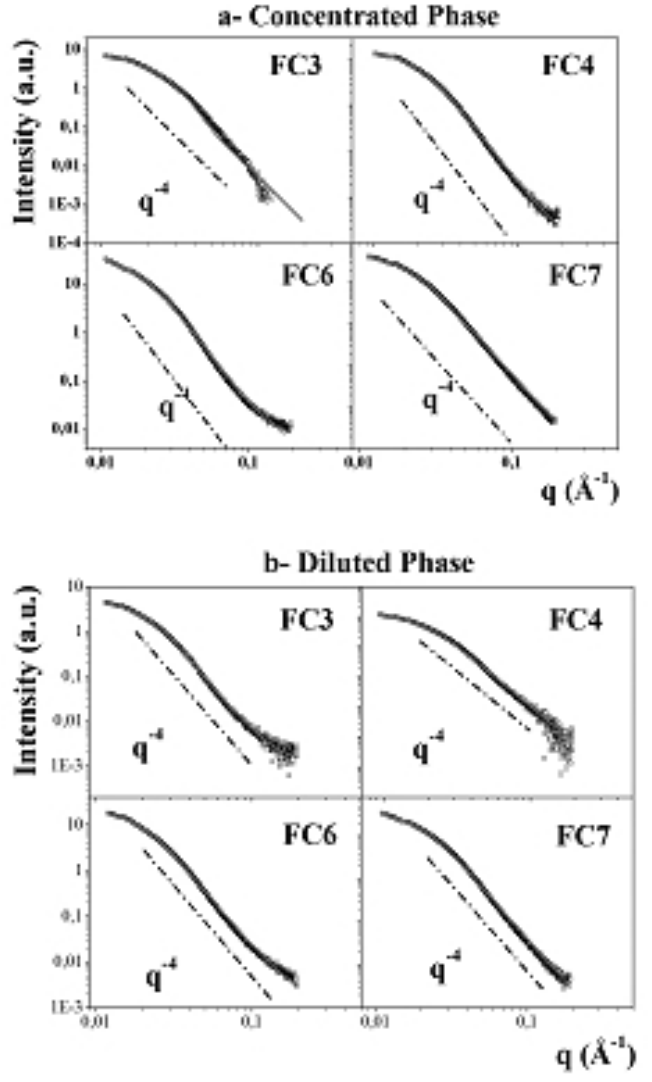

FIG. 3: SAXS curves obtained for both a) Concentrated part b) Diluted part for the samples FC3, FC4, FC6 and FC7. Open squares represent experimental data, continuous lines the fitted model according to eq. 4 , and the dash-dotted line the $q^{-4}$ behavior.

(dashed line in Fig. 3) as expected for a Porod region for noninteracting smooth nanoparticles, thus confirming that the scattering from our Laponite-ferrofluid composites is mainly due to isolated magnetic nanoparticles. This conclusion is further strengthened by our observation in Figure 4, which shows that by appropriate scaling, both the diluted and the concentrated part scattering data can be scaled onto a master curve.

The good agreement between experimental data and the model of the Global Scattering Function is shown on Figure 3 where the continuous line represents the results of the best fit obtained using equation 4 . The exponent $n$ have been fixed to 4 , the Porod value for smooth particles (here spherical) and the other parameters, $G, R_{g}$ and PDI have been left free to vary. The values of $\sigma$ were deduced from the PDI value determined using equation 3 .

Figure 5 present the values of $G, R_{0}$ and $\sigma$ deduced from the fit, both for the diluted and more concentrated parts, plotted as a function of the concentration of the number of magnetic nanoparticles per $\mathrm{cm}^{3}$ (prior to separation). The ratio of the Guinier pre-factor determined from this SAXS analyses gives the ratio between the concentrations of the two parts. The fitted values for $R_{0}$ and $\sigma$ in both cases agree well with those determined for the diluted pure ferrofluid. The values of $R_{0}$ deduced from the Guinier law are in good agreement with those obtained from the fit with the global scattering func-

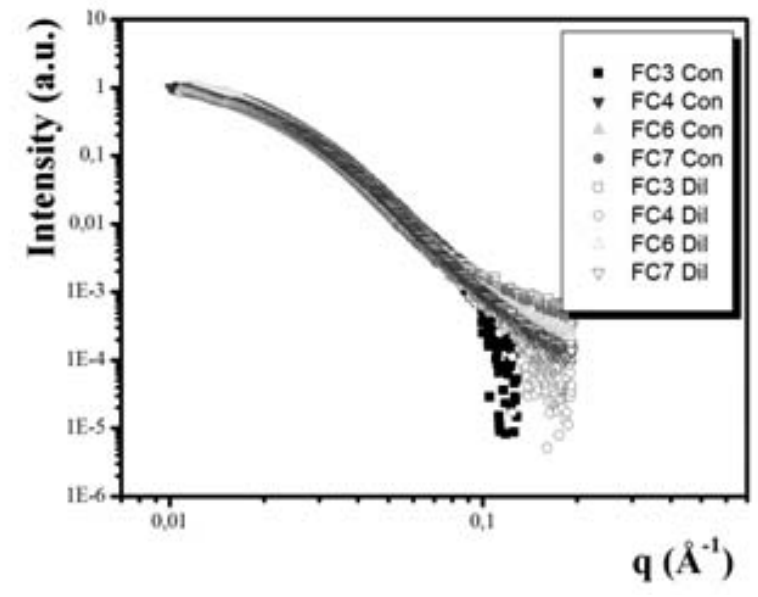

FIG. 4: Master curve for SAXS from all samples invetigated.

tion, using equation 4. Moreover, both determinations compare well with the $R_{X R}$ value using the following empirical relationship [21] $R_{X R}=R_{0} \exp \left(2.5 \sigma^{2}\right)$. It therefore confirms that for all our composite samples, the distribution of scattering object is the same for both parts of the sample, composed by spheroidal objects with a mean value of $R_{0}$ around $5.5 \mathrm{~nm}$ and a polydispersity index equal to 0.34 , close to the distribution parameters of FF sample.

We now use as form factor, a superposition of contributions of each spherical particle weighted by the square of the particle volume (see equation 6) enlightens the discussion about the FC dispersion structure. Figure 6 shows, in continuous line, the best form factor for spherical objects calculated using a log-normal function for the diluted (circle) and concentrated (square) phases of sample FC4. It indicates that the scattering intensity resembles that of an assembly of non-interacting spheres as the calculation agrees well with the experimental points. Similar results are obtained for samples FC3 to FC7 and the determined parameters of the size distribution for these samples are shown in Table 2 .

For all samples, the values of $R_{0}$ and $\sigma$, obtained in both concentrated and diluted parts, converge to the same value which compares well with the parameters of the size distribution of the pure FF sample. Moreover, these values agrees well with those deduced using the Global Scattering Function (see Figure 5). Again, this result indicates that the scattering of our colloidal mixtures of ferrite nanoparticles and Laponite nanoplatelets is mainly due to the presence in the dispersion of magnetic nanoparticles, and that in our samples, the contribution of Laponite nanoplatelets can be neglected.

As the form factor has been determined and as it coincides with that of the ferrite magnetic nanoparticles, we can now calculate the effective structure factor of the mixture which would take into account the effective interactions induced by the presence of Laponite nanoplatelets between magnetic nanoparticles. These structure factors are displayed in Figure 7 for the concentrated phases of all FC samples. They are obtained by dividing the scattering intensities of the mixtures by the form factor of our spherical magnetic nanoparticles of ferrite. The inset of Figure 7 shows for sample FC4 the comparison between the structure factors obtained in diluted and 


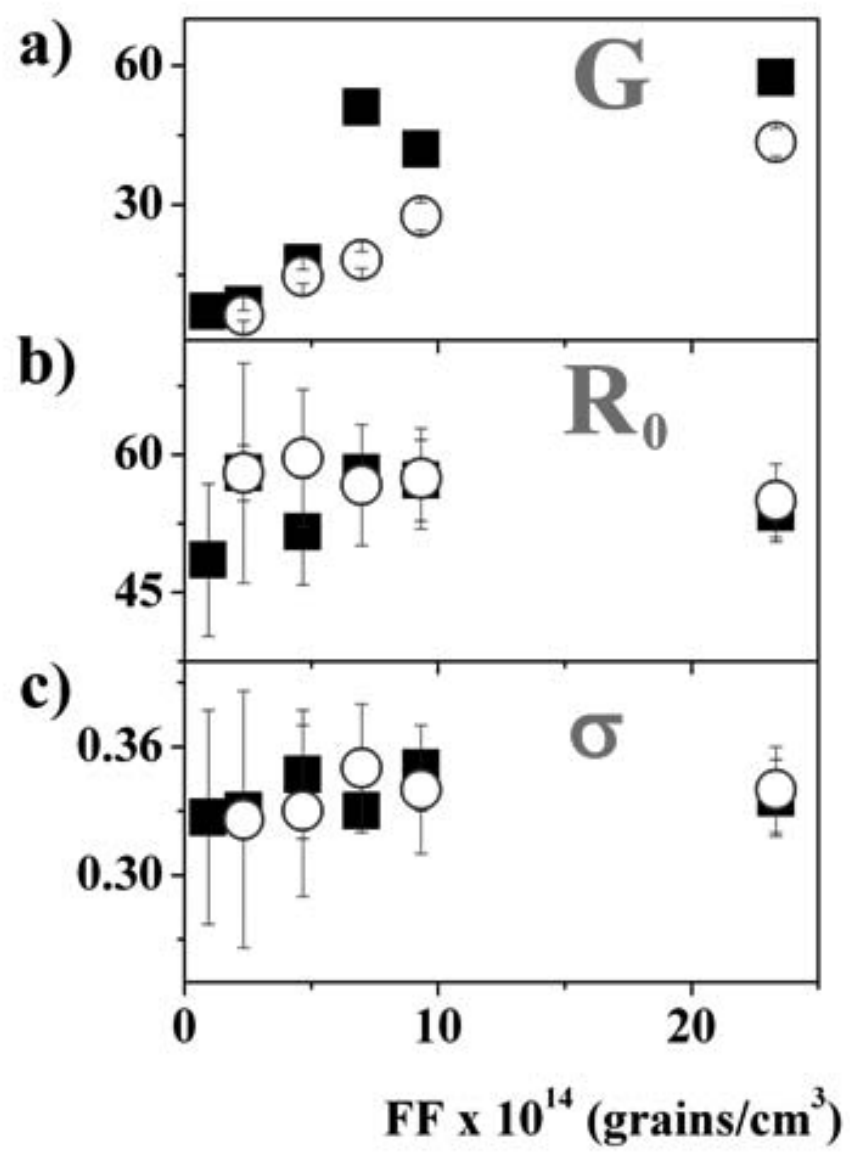

FIG. 5: Fitted parameters as a function of concentration of $F F \times$ $10^{14}$ (grains/cm3) for the FC samples listed in Table 1. Full squares for concentrated samples and open circles for the diluted ones. The values of $R_{0}$ are in units of Ångstrom.

concentrated phases.

The structure factors of Figure 7 are close to 1 over a large range of $q$ values, showing a very flat profile when $q>0.015 \AA^{-1}$ indicating that in our mixtures, the cobalt ferrite nanoparticles are not sensitive to effective attractions at short scales. Thus, on a local scale, the mixtures behave like a perfect gas of isolated magnetic nanoparticles. This is the case both for the concentrated and diluted phases. Nevertheless, for $\mathrm{q}<0.015 \AA^{-1}$ the structure factors present a slight increase, up to 1.3. A similar behavior, with a more pronounced steep rise for lower $q$ values, has recently been observed in fluid suspensions of Laponite nanoplatelets and maghemite nanoparticles [33] with low content of both kinds of nanostructures. It has been related to the existence of effective attractive interactions between magnetic nanoparticles, induced by the presence of Laponite platelets, which leads to a progressive spatial exclusion of the two kinds of nanostructures. This partial phase separation between spheres and discs cannot be excluded in this work; nevertheless such attractive effect seems to be weaker in our samples due to the lower content of both kinds of nanoparticles in the mixtures.

Our visual observations (Figure 2) also seem to indicate the existence of interactions with the presence of darker and

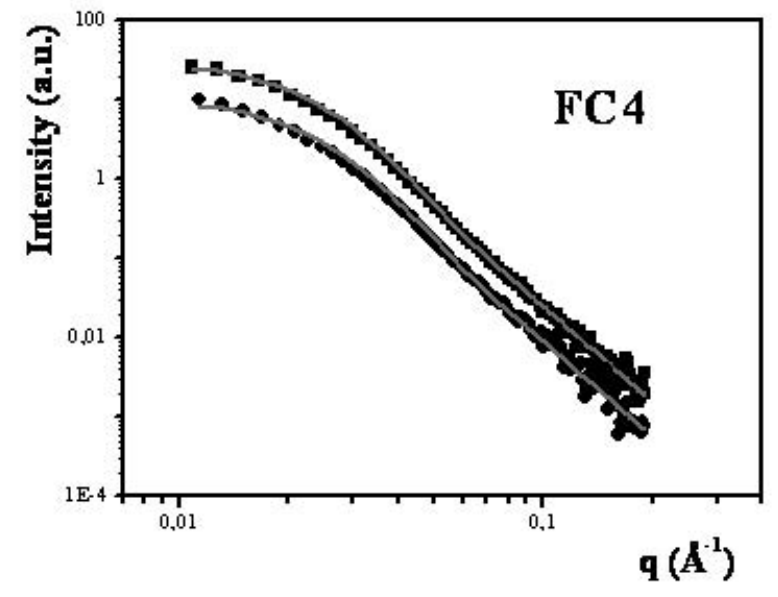

FIG. 6: SAXS curves obtained for both diluted (circle) and concentrated (square) phases of sample FC4. The full lines represent the best fit obtained with a form factor of spherical objects calculated using a log-normal function (equation 6)

\begin{tabular}{cccc}
\hline & Sample & Concentrated & Diluted \\
\hline $\mathbf{d}_{\mathbf{0}}(\mathbf{n m})$ & FC3 & 11.0 & 11.0 \\
\cline { 2 - 4 } & FC4 & 11.8 & 11.7 \\
\cline { 2 - 4 } & FC6 & 11.6 & 11.2 \\
\cline { 2 - 4 } & FC7 & 10.4 & 10.6 \\
\hline \multirow{2}{*}{$\sigma$} & FC3 & 0.33 & 0.35 \\
\cline { 2 - 4 } & FC4 & 0.34 & 0.34 \\
\cline { 2 - 4 } & FC6 & 0.36 & 0.36 \\
\cline { 2 - 4 } & FC7 & 0.35 & 0.36 \\
\hline
\end{tabular}

Table 2: Parameters of the size distribution of scattering objects obtained from fitting the scattering curves of diluted and concentrated phases accordingly to equation 6 .

lighter regions in the more concentrated phase which could be associated with a progressive segregation-like phenomenon rather than to the formation of dense aggregates. Indeed, for dense aggregates, it would be expected a structure factor with a correlation peak for large values of $q$ due to the contact between particles and a correlation hole for intermediate $q$, features which are not observed here.

In addition, we found that when submitted to a magnetic field gradient all prepared Laponite-ferrofluid composite samples show a separation in a diluted region with smaller magnetic susceptibility and a concentrated region which geometric shape is a consequence of the magnetic field lines. Also, it was observed that the composite samples return to single phase samples with apparent homogeneous visual appearance within tens of seconds after the magnet is removed. Note that this is not a quantitative study due to the inhomogeneity of the applied magnetic field generated by the disc-shaped permanent magnet which results in a net translational force. Figure 8a-e illustrates the dynamics of this separation for composite sample FC3.

Visually this behavior is very different from the one observed for the pure ferrofluid, shown in Figure metricconverterProductID8f8f, where there is no evidence for separation. This indicates that the observed separation into two different regions is due to attractive interactions between magnetic nanoparticles induced by the presence of Laponite platelets. 


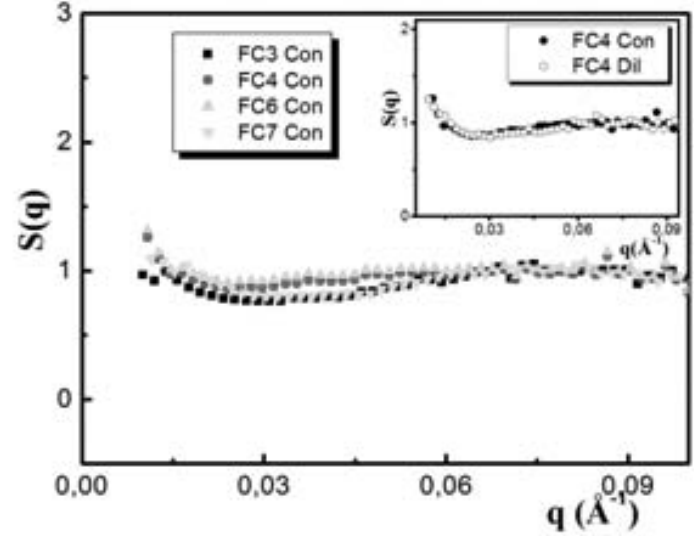

FIG. 7: Structure factor for the concentrated phase for different samples. The inset presents the results obtained for the diluted and concentrated phases of sample FC4.

\section{Magnetic Field}

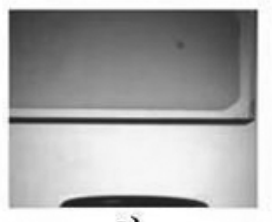

a)

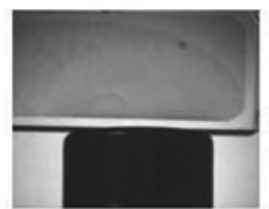

d)

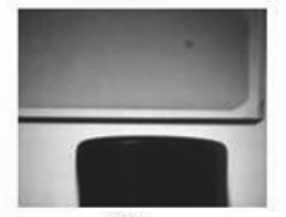

b)

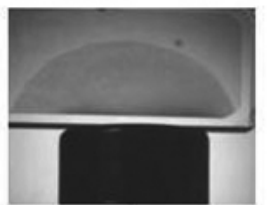

e)

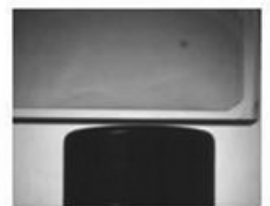

c)

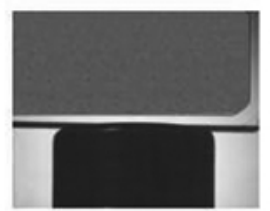

f)
FIG. 8: a)-e) Dynamics of the separation for composite sample FC3 as the rare-earth permanent magnet approach the quartz cuvette. The total elapsed time from a)-e) was about 3 minutes, and the images are evenly spaced in time. Figure metricconverterProductID8f8f shows the original FF sample under the presence of the same field.

Nevertheless, the behavior of such mixtures in the presence of an applied magnetic field would requires more SAS investigations.

\section{Conclusions}

Composite materials were obtained from the mixture of an isotropic phase Laponite solution with a diluted citrate fer- rofluid for various magnetic nanoparticle volume fractions. Such a Laponite-ferrofluid composite material raises general questions concerning self-assembly and colloidal stability. During sample mixing, the $\mathrm{pH}$ and ionic strength parameters of the final system were optimized in order to respect the range of stability of each component.

Small-angle X-ray scattering experiments were performed in order to investigate the nanostructure of the composite samples. The scattering is much more intense for the magnetic nanoparticles than for the Laponite platelets, thus the composite scattering signal is mainly due to the magnetic nanoparticles. Fitting the scattered intensities to a global scattering function including the size polydispersity and the form factor using the log-normal distribution allows us to deduce the characteristic radius and polydispersity index of the scattering object present in the composite sample, and to show that it is the same as for the pure ferrofluid sample. Using a form factor calculated by a superposition of contributions of each spherical particle weighted by the square of the particle volume assists in the discussion about the ferroclay dispersion structure. First, the fitted parameters of the size distribution of magnetic nanoparticles match well the determination with the global scattering function. Subsequently we calculate the effective structure factor of the mixture which account for the effective interactions induced by the presence of Laponite nanoplatelets between magnetic nanoparticles. The results show that on a local scale, the mixtures behave like a perfect gas of isolated magnetic nanoparticles. This is the case both for the concentrated and diluted phases. For the lower investigated values of $q$, the structure factors present a slight increase which could be associated with the existence of effective attractive interactions between magnetic nanoparticles on a longer scale, induced by the presence of Laponite platelets, which lead to a progressive spatial exclusion of the two kinds of nanostructures. Our visual observations confirm our SAS investigations.

\section{Acknowledgements}

The authors wish to acknowledge the help from the staff at beamline D11A-SAXS at the Laboratorio Nacional de Luz Sincrotron (LNLS) during the SAXS experiments. This work was supported by the Research Council of Norway (RCN) through the NANOMAT and FRINAT Programs, and by the placePlaceNameBrazilian PlaceTypeagencies PlaceTypeCAPES and CNPq.
[1] M. P. Pileni, ed. Nanocrystals Forming Mesoscopic Structures, Wiley-VCH, Weinheim, Germany, 2006 ISBN 3-527-31170$\mathrm{X}, 330$ pages.

[2] F. Bergaya, B.K.G. Theng, G. Lagaly, eds. Handbook of Clay
Science; Elsevier: London, 2006.

[3] C.Y. Matuo, F.A. Tourinho, M.H. Sousa, J. Depeyrot, A.M. Figueiredo Neto, Braz. J. Phys. 2002, 32, 458.

[4] J.N. Israelachvili, Intermolecular and Surface Forces; Aca- 
demic Press; StateplaceNew York; 1985.

[5] P.N. Pusey, J.P. Hansen, D. Levesque, J. Zinn-Justin, Liquids Freezing and Glass Transitions; North-Holland; Amsterdan; 1991.

[6] J.O. Fossum, E. Gudding, D.D.M. Fonseca, Y. Meheust, E. DiMasi, T. Gog, C. Venkataraman, Energy The International Journal, 2005, 3, 873-883.

[7] F.M. van der Kooij, H.N.W. Lekkerkerker, J. Phys. Chem. B, 1998, 102, 7829-7832

[8] D.M. Fonseca, Y. Méheust, J.O. Fossum, K.D. Knudsen and K.P.S. Parmar, Phys. Rev.E, 2009, 79, 021402.

[9] G. Meriguet, E. Dubois, M. Jardat, A. Bourdon, G. Demouchy, V. Dupuis, B. Farago, R. Perzynski, P. Turq,J. Phys.: Condens. Matter 2006, 18, 2685-S2696

[10] E.N. de Azevedo, M. Engelsberg, J.O. Fossum, and R. E. de Souza, Langmuir 2007, 23, 5100.

[11] J.O. Fossum, Y. Méheust, K.P.S. Parmar, K.D. Knudsen, K.J. Måly, D.M. Fonseca, Europhysics Letters, 2005, 74, 438-444.

[12] Baoxiang Wang, Min Zhou, Zbigniew Rozynek and Jon Otto Fossum, J.Mat.Chem. 2009, DOI:10.1039/b818502f.

[13] A. Mourchid, E. Lecolier,; H. van Damme, P. Levitz, Langmuir. 1998, 14, 4718.

[14] F.A. Tourinho, J. Depeyrot, G.J. da Silva, M.C.L. Lara, ; Braz. J. of Physics, 1998, 28 (4): 413-427.

[15] F.L.O. Paula, ; R. Aquino, G.J. da Silva, J. Depeyrot, F.A. Tourinho, J O. Fossum, K.D. Knudsen, J. Appl. Cryst. 2007, 40. 269.

[16] F.A. Tourinho, R. Franck, R. Massart, J. Mater. Sci. 1990, 25, 3249.
[17] A.F.C. Campos, F.A. Tourinho, G.J. da Silva, M.C.F.L. Lara, J. Depeyrot, Eur. Phys. J. E. 2001, 6, 29.

[18] E. Dubois, R. Perzynski, F. Bou, V. Cabuil, Langmuir. 2000, $16,5617$.

[19] J.P.C. Gabriel, C. Sanchez, P. Davidson, J. Phys. Chem. 1996, $100,11139$.

[20] H. van Olphen, An introduction to Clay Colloid Chemistry, 2nd ed. ,Krieger Publishing Company, StateplaceFlorida, 1991.

[21] F. Cousin, V. Cabuil, P.Levitz, Langmuir. 2002, 18, 1466.

[22] D.W. Thompson, T.J. Butterworth, Colloid Interface Sci., 1992, 151, 236-243.

[23] Beaucage, G.; J. Appl. Cryst, 1995, 28, 717.

[24] Schimidt, P. W.; J. Appl. Cryst, 1992, 15, 567.

[25] Debye, P.; Henderson, H. R.; Brumberger, H;.J. Appl. Phys, 1967, 28, 669.

[26] Koberstein, J. T.; Morra, B.; Stan, R. S.; J. Appl. Cryst, 1945, $13,34$.

[27] Fisher, M. E.; Burford, J. R.; Phys. Rev, 1967, 156, 593.

[28] Beaucage, G.; J. Appl. Cryst, 1996, 29, 134.

[29] Beaucage, G.; Physical Review E, 2004, 70, 031401.

[30] Beaucage, G.; J. Appl. Cryst, 2004, 37, 523.

[31] O. Glatter, Neutron, X-ray and light scattering, North Holland, Delta Series, Elsevier Science Publisher (1991).

[32] Lemaire, B.J., Panine, P., Gabriel, J.C.P., and Davidson, P.; Europhysis. Lett., 2002, 59, 55.

[33] Cousin, F., Cabuil, V., Grillo, I., Levitz, P., Langmuir, 2008, 24 11422. 